\title{
Differential Space-Time Modulation Schemes for Smart Antenna Aided Generalized Multicarrier DS-CDMA Systems
}

\author{
Bin $\mathrm{Hu}$, Lie-Liang Yang and Lajos Hanzo \\ School of ECS, University of Southampton, SO17 1BJ, UK. \\ Tel: +44-23-8059 3125, Fax: +44-23-8059 4508 \\ Email: bh202r,lly,lh@ecs.soton.ac.uk; http://www-mobile.ecs.soton.ac.uk
}

\begin{abstract}
Differential space-time modulation (DSTM) schemes were proposed for detection without any knowledge of the Channel State Information (CSI), which is an attractive feature in the presence of fast fading channels, where accurate channel estimation is a challenging task. In this contribution we invoke two DSTM schemes for improving the achievable performance in the downlink of a generalized MC DS-CDMA system employing smart antennas, namely the Differential Space-Time Spreading (DSTS) scheme and the Differential Steered Space-Time Spreading (DSSTS) scheme. In the MC DS-CDMA system considered the transmitter employs multiple antenna arrays and each of the antenna arrays consists of several antenna elements. Furthermore, the generalized MC DS-CDMA system employs both time- and frequency (TF) domain spreading, where a user-grouping technique is employed for reducing the effects of multiuser interference.
\end{abstract}

\section{INTRODUCTION}

In [1], Alamouti proposed a simple transmit diversity scheme employing a Space-Time Block Code (STBC), which was capable of achieving a substantial diversity gain by simultaneously transmitting two symbols with the aid of two antennas. Moreover, in [2], a hybrid downlink technique designed for achieving both transmit diversity and transmit beamforming was proposed for Direct Sequence Code Division Multiple Access (DSCDMA), which is referred to as Steered Space-Time Spreading (SSTS). However, the STBC scheme of [1] and the SSTS scheme of [2], as well as many other transmit diversity schemes, such as those proposed in [3], [4], [5], are based on the assumption that perfect Channel State Information (CSI) is available at the receiver. The estimation accuracy of the CSI has a grave impact on the attainable detection performance. Naturally, the estimation of these parameters increases the complexity imposed and typically requires substantial channel sounding overhead, which wastes valuable bandwidth. Furthermore, when the Channel Impulse Response (CIR) fades rapidly, its estimation based on the previous symbols might be insufficiently accurate for the reliable detection of the forthcoming symbols. By contrast, Differential Space-Time Modulation (DSTM) schemes [6], [7], [8], introduced as extensions of the traditional differential phase shift keying (DPSK) scheme, were capable of reliable data detection without any CSI, which is an attractive

The financial support of the European Union under the auspices of the Phoenix and Newcom projects and that of the EPSRC is gratefully acknowledged. feature in fast fading channels. Hence, DSTM schemes obviate the need for channel estimation at the receiver, while retaining the desirable benefits of space-time coding techniques. However, the performance of DSTM systems degrades significantly in the presence of even relatively mild interference and breaks down completely, when strong interference is inflicted [9]. To suppress the effects of co-channel interference when communicating over flat fading channels, a Differential Space-Code Modulation (DSCM) scheme was proposed by combining the merits of the DSTM and spread spectrum techniques in [9].

In this contribution, we combine the merits of DSTM and the generalized Multi-Carrier Direct Sequence Code Division Multiple Access (MC DS-CDMA) system of [10] to propose a novel Differential Space-Time Spreading (DSTS) scheme as well as a Differential Steered Space-Time Spreading (DSSTS) scheme for the sake of improving the downlink performance of generalized MC DS-CDMA systems communicating in fast fading channels. The proposed DSSTS scheme amalgamates the benefits of DSTS and beamforming. The generalized MC DSCDMA system considered includes the subclasses of both multitone DS-CDMA [11] and orthogonal MC DS-CDMA [12], [13], [14] as special cases [10]. More explicitly, the novelty of this paper is that we amalgamate the benefits of DSTS and DSSTS with those of the smart antenna aided generalized MC DS-CDMA system of [10] for the sake of improving the performance of the system in fast fading channels, while achieving both frequency and spatial diversity. Using the antenna arrays shown in Figure 1, two DSTM schemes based on the principles of DSTS and DSSTS are proposed and characterized, in order to enhance the attainable performance of the generalized MC DS-CDMA system. Furthermore, the generalized MC DSCDMA system using TF-domain spreading has been investigated, while employing a novel user-grouping technique for the sake of reducing the multiuser interference imposed.

The rest of this paper is organized as follows. In Section II the philosophies of the downlink DSTS-aided and DSSTSaided generalized Multicarrier DS-CDMA system are described and characterized. Then, Section III considers the achievable system capacity improvement of generalized MC DS-CDMA using DSTS, DSSTS and TF-domain spreading. The attainable performance of these schemes is studied comparatively in Section IV, again in the context of the generalized MC DS-CDMA system concept advocated. Finally, we provide our conclusions 
in Section V.

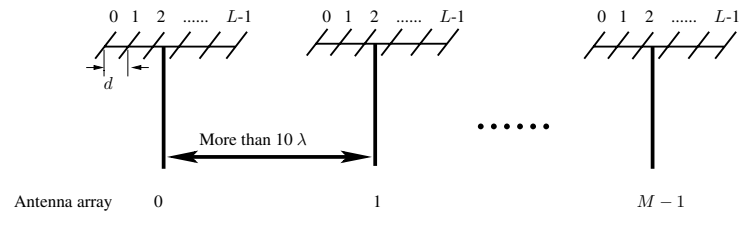

Fig. 1. Multiple transmitter antenna configuration to be used in the generalized MC DS-CDMA system considered.

\section{System Models}

In [9], a DSCM scheme was presented to suppress the interferences in flat fading channels by combining the merits of DSTM and spread spectrum techniques. It was shown that DSCM significantly outperforms DSTM in the presence of interference. Based on the DSCM technique, we extend our investigations to the DSTS and the DSSTS schemes invoked for the downlink of the generalized MC DS-CDMA system of [10]. As shown in Figure 1, the $M$ number of transmit antenna arrays are located sufficiently far apart so that the corresponding $\mathrm{MC}$ DS-CDMA signals experience independent fading. Each of the $M$ antenna arrays consists of $L$ number of elements separated by a distance of half a wavelength. When $L=1$, the standalone DSTS scheme is employed. Otherwise, the more general DSSTS scheme is invoked and hence the DSTS scheme may be viewed as a special case of the generic DSSTS scheme in conjunction with $L=1$.

\section{A. Differential Space-Time Modulation}

Before commencing our investigation of the DSTS and the DSSTS schemes, we provide a brief introduction to the unitary group code based DSTM of [7]. Let $\mathbf{X}_{n}$ be the $n$th $\left(M \times N_{l}\right)$ dimensional space-time code to be transmitted by $M$ antennas over $N_{l}$ time samples, where $N_{l}$ is the time-domain length of a space-time code. For differential space-time coding we have [7]

$$
\mathbf{X}_{n} \mathbf{X}_{n}^{H}=N_{l} \mathbf{I}_{M}, \mathbf{X}_{n}=\mathbf{X}_{n-1} \mathbf{G}_{n}, \mathbf{X}_{0}=\mathbf{D}, \forall n,
$$

where the superscript $H$ denotes the conjugate transpose operation, $\mathbf{I}_{M}$ is an $(M \times M)$-dimensional identity matrix, $\mathbf{G}_{n}$ is the matrix representing the $n$th information bearing unitary group code and $\mathbf{D}$ is a known fixed matrix. For example, for $M=N_{l}=2$, the pair

$$
\mathcal{G}=\left\{ \pm\left[\begin{array}{ll}
1 & 0 \\
0 & 1
\end{array}\right], \pm\left[\begin{array}{rr}
0 & 1 \\
-1 & 0
\end{array}\right]\right\}, \mathbf{D}=\left[\begin{array}{cc}
1 & -1 \\
1 & 1
\end{array}\right]
$$

is a unitary group code designed over the BPSK constellation $\{1,-1\}$ and $\mathbf{G}_{n} \in \mathcal{G}$. Each information bit pair in $\{00,01,10,11\}$ corresponds to an element in $\mathcal{G}$.

The received data matrix $\mathbf{Y}_{n} \in \mathcal{C}^{N_{r} \times N_{l}}$ at the output of the $N_{r}$ receive antennas has the form [7]

$$
\mathbf{Y}_{n}=\sqrt{\frac{P}{M}} \mathbf{H X}_{n}+\mathbf{N}_{n}
$$

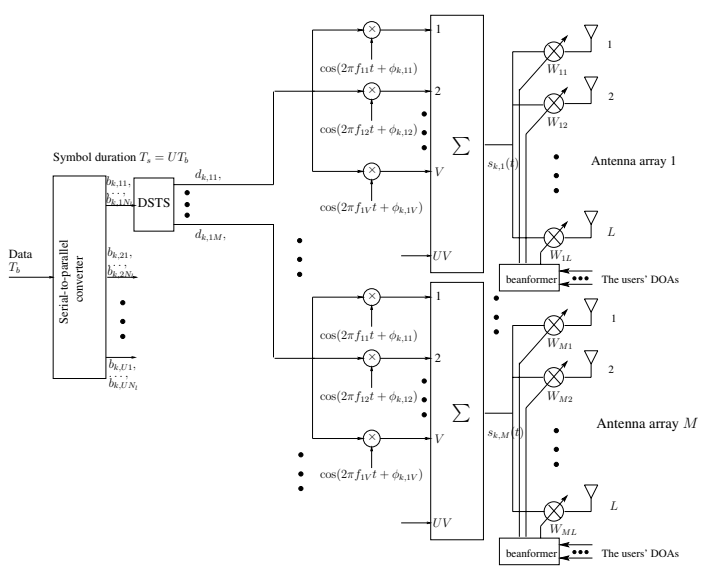

Fig. 2. The $k$ th user's transmitter schematic for the downlink of the DSSTS assisted generalized multicarrier DS-CDMA system.

where $\mathbf{H} \in \mathcal{C}^{N_{r} \times M}, \mathbf{N}_{n} \in \mathcal{C}^{N_{r} \times N_{l}}$ and $P$ denote the unknown fading matrix in a flat fading environment, the additive noise matrix and the signal-to-noise ratio (SNR) per receive antenna. Each of the elements of $\mathbf{H}$ and $\mathbf{N}_{n}$ is assumed to be independently and identically distributed complex Gaussian random variable with zero-mean and unit variance. Then, a simple differential receiver has the form of [7]

$$
\hat{\mathbf{G}}_{n}=\arg \underbrace{\max }_{\mathbf{G}_{n} \in \mathcal{G}} \operatorname{Re} \operatorname{Tr}\left\{\mathbf{G}_{n} \mathbf{Y}_{n}^{H} \mathbf{Y}_{n-1}\right\},
$$

where "ReTr" denotes the real part of the trace of a matrix.

\section{B. Transmitter Model}

The system considered in this section is an evolutionary successor of the generalized MC DS-CDMA scheme [10] using $U \cdot V$ number of subcarriers. The transmitter schematic of the $k$ th user is shown in Figure 2, where a block of $U \cdot N_{l}$ data bits each having a bit duration of $T_{b}$ is SerialParallel (S-P) converted to $U$ parallel sub-blocks. By using the mapping $\mathcal{G}$ described in Section II-A, the transmitter maps the $N_{l}$ bits of each sub-stream to the information matrix $\mathbf{G}_{k u, n}$. Then we attain the $n$th $M \times N_{l}$ space-time code $\mathbf{X}_{k u, n}=\mathbf{X}_{k u, n-1} \mathbf{G}_{k u, n}$. After spreading $\mathbf{X}_{k u, n}$ with the aid of $N_{l}$ orthogonal Walsh-Hadamard spreading codes $\left\{c_{k, 1}(t), c_{k, 2}(t), \ldots, c_{k, N_{l}}(t)\right\}, k=1,2, \ldots, K$, the $U M$ outputs of the $U$ number of DSTS blocks are multicarrier modulated by a group of subcarrier frequencies $\left\{f_{u 1}, f_{u 2}, \ldots, f_{u V}\right\}$ and then forwarded to the transmitter's $M$ arrays. The symbol duration of the DSTS signals is $N_{l} T_{s}$, and the length of the orthogonal codes is $N_{l} T_{s} / T_{c}=N_{l} N_{e}$, where $N_{e}=T_{s} / T_{c}$ and $T_{c}$ represents the chip-duration of the orthogonal spreading codes. Finally, according to the $k$ th user's Direction of Arrival (DOA), the $U M V$ signals of the $k$ th user are weighted by the transmit weight vector $\mathbf{w}_{v, m}^{(k)}$ determined for the $v$ th subcarrier of the $k$ th user, which is generated for the $m$ th array. It is reasonable to assume that the users' DOAs change slowly and hence may be estimated correctly. 
The general form of the $k$ th user's transmitted signal corresponding to the $M$ transmit antennas can be expressed as

$$
\begin{aligned}
\mathbf{s}_{k}(t)= & \sum_{u=1}^{U} \sum_{v=1}^{V} \sqrt{\frac{2 P_{k}}{V L} \frac{1}{M \cdot N_{l}}} \mathbf{W}^{k} \mathbf{X}_{k u, n} \mathbf{c}_{k} \\
& \times \cos \left(2 \pi f_{u v} t+\phi_{k, u v}\right)
\end{aligned}
$$

where $P_{k} / V$ represents the transmitted power of each subcarrier, $\mathbf{c}_{k}=\left[c_{k, 1}(t), c_{k, 2}(t), \ldots, c_{k, N_{l}}(t)\right]^{T}$ is an $N_{l}$-dimensional spreading sequences vector and $\mathbf{W}^{(k)}$ is the weight matrix, which can be expressed as

$$
\mathbf{W}^{k}=\left[\begin{array}{cccc}
\left(\mathbf{w}_{0}^{k}\right)^{*} & \mathbf{0} & \ldots & \mathbf{0} \\
\mathbf{0} & \left(\mathbf{w}_{1}^{k}\right)^{*} & \ldots & \mathbf{0} \\
\vdots & \vdots & \ddots & \vdots \\
\mathbf{0} & \mathbf{0} & \ldots & \left(\mathbf{w}_{M-1}^{k}\right)^{*}
\end{array}\right]
$$

where $\mathbf{0}=[0,0, \ldots, 0]^{T}$ is an $L$-dimensional vector, $\mathbf{w}_{m}^{k}$ is the $L$-dimensional weight vector for the $m$ th beamformer antenna array of the $k$ th user.

\section{Receiver Model}

In this paper, we consider the case of a single receive antenna. The Spatio-Temporal (ST) Channel Impulse Response (CIR) vector $h_{u v, m}^{(k)}$ between the $u v$ th subcarrier of the $k$ th user and the $m$ th antenna array can be expressed as

$$
\begin{aligned}
\mathbf{h}_{u v, m}^{(k)}(t) & =\mathbf{a}_{u v, m}^{(k)}(t) \delta\left(t-\tau_{k}\right) \\
& =\left[a_{u v, m 0}^{(k)}(t), \ldots, a_{u v, m(L-1)}^{(k)}(t)\right]^{T} \delta\left(t-\tau_{k}\right),
\end{aligned}
$$

which is an $L$-dimensional vector, where $\tau_{k}$ is the signal's delay, $a_{u v, m l}^{(k)}(t)$ is the CIR with respect to the $u v$ th subcarrier of the 1 st user and the $l$ th element of the $m$ th antenna array. Based on the assumption that the array elements are separated by half a wavelength, we can simplify $\mathbf{a}_{u v, m}^{(k)}(t)$ to

$$
\mathbf{a}_{u v, m}^{(k)}(t)=\alpha_{u v, m}^{(k)}(t) \mathbf{d}^{(k)},
$$

where $\alpha_{u v, m}^{(k)}(t)$ is the Rayleigh faded envelope's amplitude, $\quad \mathbf{d}^{(k)}=\left[1, \exp \left(j\left[\pi \sin \left(\psi^{(k)}\right)\right]\right), \ldots, \exp (j[(L-\right.$ 1) $\left.\left.\left.\pi \sin \left(\psi^{(k)}\right)\right]\right)\right]^{T}$ and $\psi^{(k)}$ is the $k$ th user's DOA.

Assuming that $K$ user signals expressed in the form of (5) are transmitted synchronously over Rayleigh fading channels, the baseband data matrix $\mathbf{Y}_{u, n} \in \mathcal{C}^{V \times N_{l} N_{e}}$ received over $V$ number of subcarriers after multicarrier demodulation can be expressed as

$$
\mathbf{Y}_{u, n}=\mathbf{H}_{u} \sum_{k=1}^{K} \sqrt{\frac{2 P_{k}}{V L} \frac{1}{M N_{l}}} \mathbf{W}^{k} \mathbf{X}_{k u, n} \mathbf{C}_{k}+\mathbf{N}_{n}
$$

where $\mathbf{N}_{n} \in \mathcal{C}^{V \times N_{l} N_{e}}$ denotes the additive white Gaussian noise (AWGN) matrix. In (9), the $V \times M L$-dimensional matrix of

$$
\mathbf{H}_{u}=\left[\begin{array}{ccc}
\left(\mathbf{h}_{u 1,0}^{(1)}(t)\right)^{T} & \ldots & \left(\mathbf{h}_{u 1,(M-1)}^{(1)}(t)\right)^{T} \\
\left(\mathbf{h}_{u 2,0}^{(1)}(t)\right)^{T} & \ldots & \left(\mathbf{h}_{u 2,(M-1)}^{(1)}(t)\right)^{T} \\
\vdots & \ddots & \vdots \\
\left(\mathbf{h}_{u V, 0}^{(1)}(t)\right)^{T} & \ldots & \left(\mathbf{h}_{u V,(M-1)}^{(1)}(t)\right)^{T}
\end{array}\right]
$$

is the unknown fading matrix, while the spreading code matrix $\mathbf{C}_{k}$ is expressed as

$$
\mathbf{C}_{k}=\left[\begin{array}{cccc}
c_{k, 1}[0] & c_{k, 1}[1] & \ldots & c_{k, 1}\left[N_{l} N_{e}-1\right] \\
c_{k, 2}[0] & c_{k, 2}[1] & \ldots & c_{k, 2}\left[N_{l} N_{e}-1\right] \\
\vdots & \vdots & \ddots & \vdots \\
c_{k, N_{l}}[0] & c_{k, N_{l}}[1] & \ldots & c_{k, N_{l}}\left[N_{l} N_{e}-1\right]
\end{array}\right] .
$$

Based on the orthogonality of the spreading codes, we multiply both sides of (9) with the spreading codes matrix $\mathbf{C}_{1}$ of the desired user and hence attain

$$
\mathbf{Z}_{u, n}=\sqrt{\frac{2 P_{1}}{V L} \frac{1}{M N_{l}}} \mathbf{H}_{u} \mathbf{W}^{1} \mathbf{X}_{1 u, n}+\mathbf{N}_{n} \mathbf{C}_{1}^{H} .
$$

In this scenario, the MRC criterion based transmit beamformer, which constitutes an effective solution to maximizing the antenna gain in the direction of the desired user, is the optimum beamformer. Let $\mathbf{w}_{m}^{k}=\mathbf{d}^{(k)}$ in (8), then the term $\mathbf{Z}_{u, n}$ can be expressed as

$$
\mathbf{Z}_{u, n}=\sqrt{\frac{2 P_{1}}{V} \frac{L}{M N_{l}}} \tilde{\mathbf{H}}_{u} \mathbf{X}_{1 u, n}+\mathbf{N}_{n} \mathbf{C}_{1}^{H},
$$

where the $(V \times M)$-dimensional matrix $\tilde{\mathbf{H}}_{u}$ is given by

$$
\tilde{\mathbf{H}}_{u}=\left[\begin{array}{ccc}
h_{u 1,0}^{(1)} & \cdots & h_{u 1,(M-1)}^{(1)} \\
h_{u 2,0}^{(1)} & \cdots & h_{u 2,(M-1)}^{(1)} \\
\vdots & \ddots & \vdots \\
h_{u V, 0}^{(1)} & \cdots & h_{u V,(M-1)}^{(1)}
\end{array}\right]
$$

Note that for $L=1$, which corresponds to the stand-alone DSTS scheme, we have $\mathbf{w}_{m}^{k}=1$. Hence no information is required about the user' DOAs by the DSTS scheme.

By invoking the differential receiver of [7], we attain the information matrix

$$
\hat{\mathbf{G}}_{k u, n}=\arg \underbrace{\max }_{\mathbf{G}_{k u, n} \in \mathcal{G}} \operatorname{Re} \operatorname{Tr}\left\{\mathbf{G}_{k u, n} \mathbf{Z}_{u, n}^{H} \mathbf{Z}_{u, n-1}\right\} .
$$

\section{CAPACITY EXTENSION USING TIME-FREQUENCY-DOMAIN SPREADING}

The Direct Sequence (DS) spreading used in MC DS-CDMA systems is carried out in the time-domain (T-domain), for example, based on orthogonal Walsh-Hadamard (WH) DS-spreading codes. However, as proposed for MC-CDMA schemes in [15], spreading in the F-domain may also be employed for the sake 
of exploiting the independent fading of the subcarriers in the F-domain. Hence in the generalized MC DS-CDMA scheme advocated, the transmitted data stream can be spread in both the T-domain and the F-domain in order to support more users, while achieving a high frequency diversity gain [16]. The resultant bandwidth is the same as that of the MC DS-CDMA scheme employing time-domain spreading only, while the total number of users supported is $V \mathcal{K}_{\max }=V N_{e}$, which is $V$ times the number of users supported by the MC DS-CDMA scheme employing time-domain only spreading.

The transmitter schematic of the broadband MC DS-CDMA system using TF-domain spreading is similar to that seen in Figure 2, except that the $V$-depth F-domain repetition of Figure 2 is now replaced by the F-domain spreading associated with an orthogonal spreading code of length $V$. Accordingly, let $\left\{c_{k}^{\prime}[0], c_{k}^{\prime}[1], \ldots, c_{k}^{\prime}[V-1]\right\}$ be the $k$ th user's orthogonal F-domain spreading code in discrete form. Following the approach of Section II, the received baseband data matrix $\mathbf{Y}_{u, n}$ can be modified from (9) to

$$
\mathbf{Y}_{u, n}=\sum_{k=1}^{K} \sqrt{\frac{2 P_{k}}{V L} \frac{1}{M N_{l}}} \mathbf{C}_{k}^{\prime} \mathbf{H}_{u} \mathbf{W}^{k} \mathbf{X}_{k u, n} \mathbf{C}_{k}+\mathbf{N}_{n}
$$

where the $(V \times V)$-dimensional F-domain spreading code matrix $\mathbf{C}^{\prime}{ }_{k}$ can be expressed as

$$
\mathbf{C}_{k}^{\prime}=\left[\begin{array}{cccc}
c_{k}^{\prime}[0] & 0 & \ldots & 0 \\
0 & c_{k}^{\prime}[1] & \ldots & 0 \\
\vdots & \vdots & \ddots & \vdots \\
0 & 0 & \ldots & c_{k}^{\prime}[V-1]
\end{array}\right]
$$

By multiplying both sides of (16) with the spreading code matrix $\mathbf{C}_{1}$ as well as $\mathbf{C}_{1}^{\prime}$ of the desired user and letting $\mathbf{w}_{m}^{k}=$ $\mathbf{d}^{(k)}$, we have

$$
\begin{aligned}
\mathbf{Z}_{u, n}= & \sqrt{\frac{2 P_{1}}{V} \frac{L}{M N_{l}}} \tilde{\mathbf{H}}_{u} \mathbf{X}_{1 u, n}+\mathbf{C}_{1}^{\prime H} \mathbf{N}_{n} \mathbf{C}_{1}^{H} \\
& +\sum_{k=2}^{K^{\prime}} \sqrt{\frac{2 P_{k}}{V L} \frac{1}{M N_{l}}} \mathbf{C}_{1}^{\prime H} \mathbf{C}_{k}^{\prime} \mathbf{d}^{(1)^{T}} \mathbf{d}^{(k)^{*}} \tilde{\mathbf{H}}_{u} \mathbf{X}_{k u, n},
\end{aligned}
$$

where $K^{\prime}$ is the number of users sharing the same T-domain spreading code matrix with the desired user, while the term $\sum_{k=2}^{K^{\prime}} \sqrt{\frac{2 P_{k}}{V L} \frac{1}{M N_{l}}} \mathbf{C}^{\prime H}{ }_{1}^{H} \mathbf{C}_{k}^{\prime} \mathbf{d}^{(1)^{T}} \mathbf{d}^{(k)^{*}} \tilde{\mathbf{H}}_{u} \mathbf{X}_{k u, n}$ is caused by the Multiuser Interference (MUI).

We know from Equation (18) that multiuser interference is inevitably introduced, since the orthogonality of the F-domain spreading codes cannot be retained over frequency-selective fading channels. However, the desired signal is not interfered by the transmitted signals of the users employing different orthogonal T-domain spreading codes, provided that synchronous transmission of all the $K^{\prime} \mathcal{K}_{\max }$ user signals as well as flatfading of each subcarrier are assumed. Only the users sharing the same T-domain spreading codes matrix with the desired user will impose interference on the desired user. Therefore, the MUI term can be reduced, if we carefully select the
$K^{\prime}-1$ users, namely those which have the lowest interference coefficient with respect to the desired user, from the set of all the $K^{\prime} \mathcal{K}_{\max }$ users for the sake of sharing the same T-domain spreading code with the desired user. Let the interference coefficient be defined as $\mathbf{d}^{(1)^{T}} \mathbf{d}^{(k)^{*}}$, which can be evaluated before transmission based on the assumption that the users' DOAs are perfectly estimated. Following this user-grouping procedure, the effect of the interfering signals imposed on the desired user's signal becomes less pronounced. Therefore, the achievable BER performance is improved. Note that in the standalone DSTS scheme no beamforming is invoked and hence the interference coefficient becomes as high as $\mathbf{d}^{(1)^{T}} \mathbf{d}^{(k)^{*}} \equiv 1$. Therefore, the achievable BER performance cannot be improved in the DSTS scheme by employing the above-mentioned user-grouping procedure.

\section{PERformance Results}

In this section the BER performance of the downlink of a generalized MC DS-CDMA system employing both DSTS and DSSTS, using 32-chip WH codes as T-domain spreading sequences as well as 4-chip WH codes as F-domain spreading codes is studied. Perfect power control is assumed, implying that we have $P_{0}=P_{1}=\ldots=P_{K}$.

In Figure 3 we use three different types of antenna arrays, namely a $(2 \times 2)$-dimensional antenna array $(M=2, L=2)$ corresponding to the DSSTS scheme, a $(4 \times 1)$-dimensional antenna array $(M=4, L=1)$ and a $(2 \times 1)$-dimensional antenna array ( $M=2, L=1$ ) corresponding to the stand-alone DSTS scheme. In these investigations $K=4$ users are supported. Observe in Figure 3 that as expected, the attainable performance of the DSTS and the DSSTS schemes is about $3 \mathrm{~dB}$ worse than those of the STS and the SSTS schemes, respectively. The DSTS system using a $(4 \times 1)$-dimensional antenna array ( $M=4, L=1$ ) achieved a slightly better performance than the DSTS system using a $(2 \times 1)$-dimensional antenna array ( $M=2, L=1)$, since the highest incremental diversity gains are achieved upon increasing the number of antennas from one to two antennas, i.e. by $100 \%$, while adding further additional antennas achieves modest extra diversity gains on the downlink. Furthermore, Figure 3 shows that the DSSTS system employing a $(2 \times 2)$-dimensional antenna array $(M=2, L=2)$ outperforms the DSTS system using a $(4 \times 1)$-dimensional antenna array $(M=4, L=1)$, suggesting that the DSSTS scheme employs DSTS for the sake of obtaining transmit diversity, but additionally invokes beamforming to attain a higher SNR gain.

In Figure 4, generalized MC DS-CDMA systems supporting $K=1,32$ and 64 users were studied. It transpires from Figure 4 that the performance of the system supporting 32 users is similar to that of the system serving a single user. Recall the assumptions of using orthogonal multicarrier signals, synchronous transmission of the $K$ user signals as well as slow flat-fading of each subcarrier, where no interference is encountered between the 32 users employing different orthogonal 32chip Walsh codes as T-domain DS spreading codes. In order 
to extend the user-capacity of the system, TF-domain spreading is employed for the sake of supporting 64 users. Consequently, multiuser interference among the users sharing the same T-domain spreading code is inevitably introduced, since the orthogonality of the F-domain spreading codes cannot be retained, when communicating over frequency-selective fading channels. Then a specific user grouping technique is employed by the DSSTS system for the sake of reducing the multiuser interference imposed. Figure 4 demonstrates that the performance of the DSTS system supporting 64 users is significantly worse than that supporting a single user or even 32 users. By contrast, the DSSTS system supporting 64 users and employing the above-mentioned interference coefficient based user grouping technique achieves a slightly worse BER performance than that serving a single user, nonetheless, attaining a $5.7 \mathrm{~dB}$ SNR gain compared to the DSTS system at a BER of $10^{-5}$.

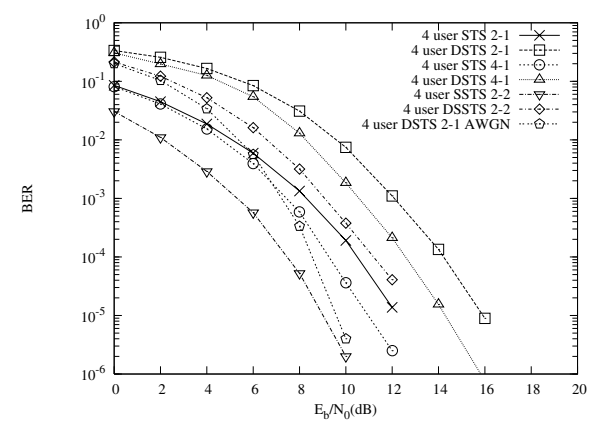

Fig. 3. BER versus SNR performance of the downlink of a generalized MC DS-CDMA wireless system using 32-chip Walsh-Hadamard (WH) codes as T-domain spreading sequences and 4-chip WH codes as F-domain spreading sequences, employing three different types of antenna arrays, namely a $(2 \times 2)$ dimensional antenna array $(M=2, L=2)$ corresponding to the DSSTS scheme, a $(4 \times 1)$-dimensional antenna array $(M=4, L=1)$ and a $(2 \times$ 1)-dimensional antenna array $(M=2, L=1)$ corresponding to the DSTS scheme.

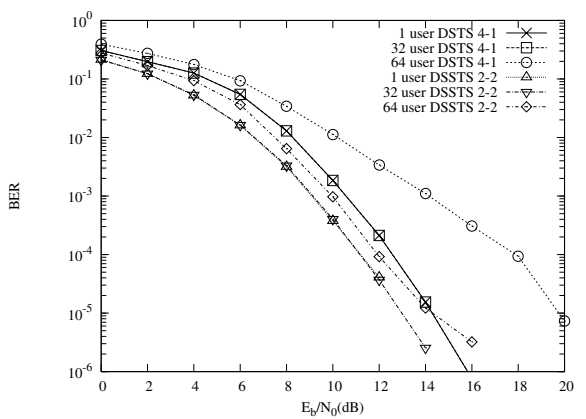

Fig. 4. BER versus SNR performance of the downlink of a generalized MC DS-CDMA wireless system using 32-chip WH codes as T-domain spreading sequences and 4-chip WH codes as F-domain spreading sequences. TF-domain spreading was used to extend the capacity of the DSTS and the DSSTS systems, while an interference coefficient based user grouping technique was used in the DSSTS system to reduce the interference between the users sharing the same 32-chip WH code as T-domain spreading code. Two types of antenna arrays were employed, namely a $(2 \times 2)$-dimensional antenna array $(M=2, L=2)$, and a $(4 \times 1)$-dimensional antenna array $(M=4, L=1)$.

\section{CONCLUSIONS}

In this contribution we have proposed two differential spacetime processing schemes based on the principles of DSTS and DSSTS, in order to enhance the attainable performance of generalized MC DS-CDMA systems operating in fast fading channels. As expected, the attainable performance of both the DSTS and DSSTS schemes is about $3 \mathrm{~dB}$ worse than those of the corresponding non-differential STS and SSTS schemes, respectively, which can be achieved however without any CSI, i.e. at a reduced complexity. The DSSTS system employing a $(2 \times 2)$-dimensional antenna array $(M=2, L=2)$ outperforms the DSTS system using a $(4 \times 1)$-dimensional antenna array $(M=4, L=1)$, suggesting that the DSSTS scheme employs DSTS for the sake of obtaining transmit diversity, but additionally also invokes beamforming to attain a higher SNR gain. Furthermore, generalized MC DS-CDMA using TF-domain spreading has been investigated and a novel user-grouping technique based on the interference coefficients was proposed for reducing the multiuser interference.

\section{REFERENCES}

[1] S. M. Alamouti, "A simple ransmit diversity technique for wireless communications," IEEE Journal on Selected Areas in Communications, vol. 16, no. 8, pp. 1451-1458, Oct. 1998.

[2] Robert A. Soni and R. Michael Buehrer and Roger D. Benning, "Intelligent Antenna System for cdma2000," IEEE Signal Processing Magazine, pp. 54-67, July 2002.

[3] Proposed TDOC: 662/98 to ETSI SMG2 UMTS Standards, Space-time block coded transmit antenna diversity for WCDMA, December 1998.

[4] Y. Zhou and F. Chin and Y. C. Liang and C. C. Ko, "Performance Comparison of Transmit diversity and Beamforming for the Downlink of DS-WCDMA System," IEEE Transactions on Wireless Communications, vol. 2, no. 2, pp. 320-334, Mar. 2003.

[5] Y. C. Liang and F. Chin, "Transmit antenna array techniques for celluar WCDMA systems," Proc. IEEE 9th PIMRC, pp. 1396-1400, Sept. 1998.

[6] V. Tarokh and H. Jafarkhani, "A differential detection scheme for transmit diversity," IEEE Journal on Selected Areas in Communications, vol. 18, pp. 1169-1174, July 2000

[7] B. L. Hughes, "Differential space-time modulation," IEEE Transactions on Information Theory, vol. 46, pp. 2567-2578, Nov. 2000.

[8] B. M. Hochwald and W. Sweldens, "Differential unitary space-time modulation," IEEE Transactions on Communications, vol. 48, pp. 2041-2052, Dec. 2000.

[9] J. H. Liu and J. Li and H. B. Li and E. G. Larsson, "Differential spacecode modulation for interference suppression," EEE Transactions on Signal Processing, vol. 49, pp. 1786-1795, Aug. 2001.

[10] L.-L. Yang and L. Hanzo, "Performance of generalized multicarrier DSCDMA over Nakagami- $m$ fading channels," IEEE Transactions on Communications, vol. 50, pp. 956 - 966, June 2002.

[11] L. Vandendorpe, "Multitone spread spectrum multiple access communications system in a multipath Rician fading channel," IEEE Transactions on Vehicular Technology, vol. 44, no. 2, pp. 327-337, 1995.

[12] L. Hanzo, L. L. Yang, E. L. Kuan, and K. Yen, Single- and Multi- Carrier DS-CDMA. John Wiley \& Sons - IEEE Press, 2003, 1060 pages.

[13] L. Hanzo, M. Munster, B. J. Choi, and T. Keller, OFDM and MC-CDMA. John Wiley \& Sons - IEEE Press, 2003, 960 pages.

[14] E. A. Sourour and M. Nakagawa, "Performance of orthogonal multicarrier CDMA in a multipath fading channel," IEEE Transactions on Communications, vol. 44, pp. 356-367, March 1996.

[15] Ramjee Prasad and Shinsuke Hara, "Overview of Multicarrier CDMA," IEEE Communications Magazine, pp. 126-133, Dec. 1997.

[16] L.-L. Yang and L. Hanzo, "Performance of Broadband Multicarrier DSCDMA Using Space-Time Spreading-Assisted Transmit Diversity," in IEEE Transactions on Wireless Communications, vol. 4, pp. 885-894, May 2005. 